\title{
NILPOTENT SUBGROUPS OF THE GROUP OF FIBRE HOMOTOPY EQUIVALENCES
}

\author{
Y. FÉlix and J. C. Thomas*
}

\begin{abstract}
Let $\xi=(E, p, B, F)$ be a Hurewicz fibration. In this paper we study the space $\mathcal{L}_{G}(\xi)$ consisting of fibre homotopy self equivalences of $\xi$ inducing by restriction to the fibre a self homotopy equivalence of $F$ belonging to the group $G$. We give in particular conditions implying that $\pi_{1}\left(\mathcal{L}_{G}(\xi)\right)$ is finitely generated or that $\mathcal{L}_{1}(\xi)$ has the same rational homotopy type as $\operatorname{aut}_{1}(F)$.
\end{abstract}

Let $\xi=(E, p, B, F)$ be a Hurewicz fibration where $B$ and $F$ are compactly generated spaces. The set of free (not necessarily fibre) homotopy classes of free fibre homotopy equivalences of $\xi$ into itself is a group $\mathcal{L}(\xi)$, for the multiplication induced by the composition of maps.

Recall that a fibre homotopy equivalence $f: E \rightarrow E$ induces an homotopy equivalence of $p^{-1}(b)$ for each $b \in B$ (A theorem of Dold ([4, Theorem 6.3]) asserts that the converse is true if $B$ is a CW complex). There exists thus a natural map

$$
R: \mathcal{L}(\xi) \longrightarrow \text { Aut } F,
$$

where Aut $F$ denotes the group of free homotopy classes of free homotopy equivalences of the space $F$ into itself.

Our purpose in this paper is the study of the groups $\mathcal{L}_{G}(\xi)=R^{-1}(G)$ and the spaces $L_{G}(\xi)$ where $G$ is some subgroup of Aut $F$. Here aut $X$ is the monoid of free homotopy equivalences of the space $X$ into itself, $\operatorname{aut}_{G} X$ is the submonoid of aut $X$ consisting of the path components belonging to $G$, and $L_{G}(\xi)$ is the space of fibre homotopy self-equivalences of $\xi$ inducing by restriction to the fibre an element of $\operatorname{aut}_{G} F: \pi_{0}\left(L_{G}(\xi)\right)=\mathcal{L}_{G}(\xi)$.

*Partially supported by a CNRS-CGRI-FNRS agreement 
When $G$ is reduced to the identity $\{1\}$, we obtain the (connected) monoid aut $F$ of self-equivalences homotopic to the identity, and the monoid $L_{1}(\xi)$ of fibre self-equivalences of $\xi$ inducing by restriction to the fibre a map homotopic to the identity.

The monoids $L_{G}(\xi)$ and $\operatorname{aut}_{G} F$ are H-spaces, so that all their components have the same homotopy type. The study of the homotopy type of $L_{G}(\xi)$ is therefore reduced to the consideration of

(a) the map $\pi_{0}(R): \pi_{0}\left(L_{G}(\xi)\right)=\mathcal{L}_{G}(\xi) \rightarrow \pi_{0}\left(\operatorname{aut}_{G} F\right)=G$. and

(b) the restriction map $R_{1}: L_{1}(\xi) \rightarrow$ aut $_{1} F$.

Our main problems can be stated as follows :

1. On what conditions is the group $\mathcal{L}_{1}(\xi)$ finitely generated or finite (rigidity of the fibration) [cf. Theorem 4, below].

2. On what conditions is the map $R_{1}$ a homotopy equivalence [cf. for instance Theorem 5 below].

We first show that the group $\mathcal{L}_{1}(\xi)$ and the groups $\pi_{i}\left(L_{1}(\xi)\right), i \geq 1$, are finitely generated groups when the base $B$ is a simply connected finite CW complex and the fibre $F$ has the homotopy type of a simply connected finite type CW complex. In the particular case the base is a sphere, the result is more precise. We have indeed :

Theorem 1. If $\xi=\left(E, p, S^{n}, F\right)$ is a fibration with clutching function $\alpha: S^{n-1} \rightarrow \operatorname{aut}_{G} F$, then there exists an exact sequence of groups

$$
\pi_{1}\left(\operatorname{aut}_{G} F\right) \stackrel{\partial_{\alpha}}{\longrightarrow} \pi_{n}\left(\operatorname{aut}_{G} F\right) \stackrel{L}{\longrightarrow} \mathcal{L}_{G}(\xi) \stackrel{\pi_{0}(R)}{\longrightarrow} G_{\alpha} \rightarrow 1
$$

where

(1) $\partial_{\alpha}$ is the Samelson product by $\{\alpha\} \in \pi_{n-1}\left(\operatorname{aut}_{G} F\right)$.

(2) $G_{\alpha}$ is the stabilizer of $\{\alpha\}$ in $G$ for the natural action of $G$ on $\pi_{n-1}\left(\operatorname{aut}_{G} F\right), G_{\alpha}=\{g \in G \mid g \cdot \alpha=\alpha\}$.

In case $G=$ Aut $X$, this result has been obtained by K. Tsukiyama $([\mathbf{2 1}])$, as a corollary of a result of D. Gottlieb ([9]). Theorem 1 is obtained in a similar way from a slight modification of the quoted result of D. Gottlieb.

The interest of the above generalization of Tsukiyama's result lies in

Theorem 2. Under the hypothesis of Theorem 1, if we suppose that $F$ is a nilpotent space and that $G$ acts unipotently on each $H_{i}(F ; \mathbb{Z})$, then $\mathcal{L}_{G}(\xi)$ is a nilpotent group. 
Theorem 2 follows from Theorem 1 and Theorem 3.3 of $([\mathbf{6}])$. Indeed, Theorem 3.4 of $([\mathbf{6}])$ states that under our conditions the group $G$ is nilpotent.

As a consequence of Theorem 2, we obtain after 0-localization the exact sequence

$$
\pi_{1}\left(\operatorname{aut}_{G} F\right) \otimes \mathbb{Q} \stackrel{\partial_{\alpha} \otimes \mathbb{Q}}{\longrightarrow} \pi_{n}\left(\operatorname{aut}_{G} F\right) \otimes \mathbb{Q} \stackrel{L \otimes \mathbb{Q}}{\longrightarrow} \widehat{\mathcal{L}_{G}(\xi)} \stackrel{\widehat{R}}{\rightarrow} \widehat{G_{\alpha}} \rightarrow 1,
$$

where $\widehat{\mathcal{L}_{G}(\xi)}$ and $\widehat{G_{\alpha}}$ respectively denote the Malcev completions of the nilpotent groups $\mathcal{L}_{G}(\xi)$ and $G_{\alpha}$.

Our next result gives a complete description of this exact sequence in terms of a Sullivan model of $F$ (see $([\mathbf{2 0}],[\mathbf{1 1}])$ for basic notions in rational homotopy theory).

Let $(\wedge X, d)$ be a minimal model for $F$ with a fixed K.S. basis $\left(x_{i}\right)_{i \in I}$. A derivation $\theta$ of $(\wedge X, d)$ is locally nilpotent (rel. $\left.\left(x_{i}\right)\right)$ if we have

$$
\theta\left(x_{i}\right) \in \wedge\left(\bigoplus_{j<i} x_{j} \mathbb{Q}\right) .
$$

Denote by $\operatorname{Der}_{*} \wedge X$ the graded Lie algebra of derivations of $(\wedge X, d)$. This is a $\mathbb{Z}$-graded Lie algebra. The differential $D=[d,-]$ makes Der $_{*} \wedge X$ into a graded differential Lie algebra. We define the sub differential Lie algebra $L_{*}$ by :

$$
\begin{array}{ll}
L_{-i}=\operatorname{Der}_{-i}(\wedge X), & i \geq 1 \\
L_{j}=0 & j \geq 1 \\
L_{0} & \text { is the subspace of } \operatorname{Der}_{0}(\wedge X) \text { consisting of cycles } \\
& \text { which are locally nilpotent with respect to the } \\
& \text { fixed K.S. basis. }
\end{array}
$$

Theorem 3. Let $\xi=\left(E, p, S^{n}, F\right)$ be a unipotent fibration with fibre a nilpotent space $F$, and let $G$ be a maximal subgroup of Aut $F$ acting unipotently on $H_{*}(F ; \mathbb{Z})$. If $G$ is torsion free, then we have the exact sequence

$$
H_{-1}\left(L_{*}, D\right) \stackrel{\partial_{\eta}}{\longrightarrow} H_{-n}\left(L_{*}, D\right) \stackrel{\lambda}{\longrightarrow} \widehat{\mathcal{L}_{G}(\xi)} \stackrel{\rho}{\longrightarrow} \exp \left(H_{0}\left(L_{*}, D\right)_{\eta}\right) \rightarrow 1,
$$

where

(a) $\eta$ is a derivation of degree $(-n+1)$ which is determined by the classifying map $k$ of $\xi$. Moreover $D(\eta)=0$.

(b) $\partial_{\eta}$ is the Lie bracket by the homology class of $\eta$.

(c) $H_{0}\left(L_{*}, D\right)_{\eta}=\left\{\gamma \in H_{0}\left(L_{*}, D\right) \mid[\gamma, \eta]=0\right\}$.

(d) $\exp \left(H_{0}\left(L_{*}, D\right)_{\eta}\right)$ denotes the Malcev group associated to the locally nilpotent Lie algebra $H_{0}\left(L_{*}, D\right)_{\eta}$. 
Note that the torsion free hypothesis on $G$ is not difficult to satisfy. For instance, if $X$ is a rational space, then Aut $X$ is a torsion free group ([3, Theorem 2.5]).

On the other hand, if $X$ is a finite type virtually nilpotent CW complex, then Aut $X$ is finitely generated $([\mathbf{5}])$.

Using rational homotopy, we can make precise the structure of $\mathcal{L}_{G}(\xi)$ in two interesting cases.

It is well known that fibrations $\xi$ with fibre an homogeneous space $K / H$ with $\operatorname{rank} K=\operatorname{rank} H$ have special properties. We know that the Serre spectral sequence of $\xi$ with rational coefficients collapses at the $E_{2}$-term. Here we show that the space of self-equivalences of $\xi$ is very small. More precisely,

Theorem 4. Let $\xi:(E, p, B, F)$ be a fibration where all spaces are simply connected and of the homotopy type of finite $C W$ complexes. We suppose that $F$ is an homogeneous space, $F=K / H$ with $K$ and $H$ compact connected Lie groups of the same rank, and that $H^{2 n+1}(B ; \mathbb{Z})$ is a finite group for $n \geq 0$. Let $G$ be a maximal subgroup of Aut $F$ acting unipotently on $H_{*}(F ; \mathbb{Z})$.

Then,

(a) the group $\mathcal{L}_{G}(\xi)$ is a finite group.

(b) the space $L_{1}(\xi)$ is a connected finite dimension $H$-space, and for $n>1$, we have

$$
\operatorname{dim} . \pi_{2 n-1}\left(L_{1}(\xi)\right) \otimes \mathbb{Q}=\sum_{p \leq n} \operatorname{dim} . H^{2 p}(B ; \mathbb{Q}) \otimes \pi_{2 n-2 p}\left(B_{\mathrm{aut}_{1} F}\right) .
$$

Remark that (a) means that two self-equivalences of $\xi$ inducing homotopic restrictions to the fibre $F$ localized at 0 are already homotopic, after localization at 0 .

In a similar way, we obtain

Theorem 5. Let $\xi:(E, p, B, F)$ be a fibration where all spaces are simply connected and of the homotopy type of finite $C W$ complexes. We suppose that there exists an integer $n$ such that $\pi_{q}(F)$ is finite for $q>n$ and $\tilde{H}^{q}(B ; \mathbb{Z})$ is finite for $q \leq n$. Let $G$ be a maximal subgroup of Aut $F$ acting unipotently on $H_{*}(F ; \mathbb{Z})$. Then,

(a) The restriction map $\hat{R}: \widehat{\mathcal{L}_{G}(\xi)} \rightarrow \hat{G}$ is injective. This implies that $L_{1}(\xi)$ is a connected $H$-space.

(b) The restriction $R$ induces a rational homotopy equivalence

$$
L_{1}(\xi) \rightarrow \operatorname{aut}_{1} F
$$




\section{Proof of Theorem 1}

We consider the fibre sequence

$$
\operatorname{aut}^{\bullet} X \rightarrow \text { aut } X \stackrel{e}{\rightarrow} X
$$

where $e$ is the evaluation map. Taking the classifying space of the monoids aut $X$ and aut $X$, we get a fibration sequence (up to homotopy)

$$
\mathcal{U}: X \rightarrow B_{\text {aut }} \cdot X \stackrel{u}{\rightarrow} B_{\text {aut } X}
$$

which is universal for Hurewicz fibrations with fibre $X,([6$, Proposition 4.1]).

By analogy with the theory of fibre bundles, we consider Aut $F$ as the "structural group" of a Hurewicz fibration $\xi=(E, p, B, F)$ and we shall say that the structural group of $\xi$ can be reduced to $G \subset$ Aut $F$ if $\xi$ admits a classifying map $k: B \rightarrow B_{\text {aut } F}$ such that the image of the map $\pi_{1}(k): \pi_{1}(B) \rightarrow \pi_{1}\left(B_{\text {aut } F}\right) \cong \pi_{0}($ aut $F)$ is contained in $G$. This is only a useful analogy because the classifying map does not factor at all through the classifying space $B_{G}$. In fact we can form the monoid aut $_{G} F$ of self-equivalences of $F$ whose homotopy classes belong to $G$. In case of a $G$-reduction the classifying map $k$ factors through the space $B_{\mathrm{aut}_{G} F}([\mathbf{1 7}],[6$, Proposition 4.2]). The fibration

$$
\left(\mathcal{U}_{G}\right): F \rightarrow B_{\text {aut }_{G}^{\bullet} F} \rightarrow B_{\text {aut }_{G} F}
$$

is a universal fibration for fibrations with fibre $F$ whose "structural group" can be reduced to $G$.

Example. Let $B=S^{n}$. A Hurewicz fibration $\xi=(E, p, B, F)$ is determined, up to fibre homotopy, by the homotopy class $\{\alpha\}$ of a clutching function $\alpha: S^{n-1} \rightarrow$ aut $F$. In this case the structural group of $\xi$ can be reduced to $G$ if and only if for some point $p$ in $S^{n-1}$ the class $[d(p)]$ belongs to $G$.

Henceforth we shall fix a Hurewicz fibration $\xi=(E, p, B, F)$ whose base is a CW complex and with classifying map $k: B \rightarrow B_{\text {aut }_{G} F}$.

Because Hurewicz fibrations give rise to a homotopy functor $([\mathbf{1}])$, and from $([\mathbf{1 9}$, Chapitre 7 , Section 7 , Theorem 11]), we can choose $k$ as an inclusion and $\xi$ as the restriction of $\left(\mathcal{U}_{G}\right)$ to $B$.

Let $L^{*}\left(\xi, \mathcal{U}_{G}\right)$ be the space of fibre preserving maps from $E$ to $B_{\text {aut }}^{\bullet} F$ which carry each fibre of $\xi$ into a fibre of $\mathcal{U}_{G}$ by a homotopy equivalence. Let $L^{*}\left(\xi, \mathcal{U}_{G} ; k\right)$ be the set of maps in $L^{*}(\xi, \mathcal{U})$ with the additional property that every map $f \in L^{*}(\xi, \mathcal{U})$ covers a map $B \rightarrow B_{\text {aut }_{G} F}$ which is homotopic to $k$. 
We denote by $L\left(B, B_{\text {aut }_{G} F} ; k\right)$ the component of $k$ in the space of maps from $B$ to $B_{\text {aut }_{G} F}$ and by

$$
\Phi: L^{*}\left(\xi, \mathcal{U}_{G} ; k\right) \rightarrow L\left(B, B_{\mathrm{aut}_{G} F} ; k\right)
$$

the map that associates to every $f \in L^{*}\left(\xi, \mathcal{U}_{G}\right)$ the map $g \in L\left(B, B_{\text {aut }_{G} F}\right)$ covered by $f$.

Following the lines of the proof given by D. Gottlieb in the case $B_{\text {aut }} F$ $([\mathbf{9}$, Theorem 1$])$, we obtain

Proposition 1. Let $F \rightarrow E \rightarrow B$ be a fibration whose base is a $C W$ complex and with classifying map $k$. If $\Phi$ is defined as above, then :

(1) $\Phi^{-1}(k) \cong L_{G}(\xi)$.

(2) $L_{G}(\xi) \rightarrow L^{*}\left(\xi, \mathcal{U}_{G} ; k\right) \stackrel{\Phi}{\longrightarrow} L\left(B, B_{\text {aut }_{G} F} ; k\right)$ is a principal fibration with a left action of $L_{G}(\xi)$ on $L^{*}\left(\xi, \mathcal{U}_{G}\right)$ given by composition of maps.

(3) If $E$ is compactly generated, then $\pi_{i}\left(L^{*}\left(\xi, \mathcal{U}_{G} ; k\right)\right)$ is trivial for all $i \geq 0$.

This implies immediately :

Corollary 1. If E is compactly generated, then

$$
\mathcal{L}_{G}(\xi)=\pi_{0}\left(L_{G}(\xi)\right)=\pi_{1}\left(L\left(B, B_{\operatorname{aut}_{G} F} ; k\right)\right) .
$$

and

$$
\pi_{i}\left(L_{G}(\xi)\right) \cong \pi_{i+1}\left(L\left(B, B_{\operatorname{aut}_{G} F} ; k\right)\right), \quad i \geq 1
$$

In the particular case when $B=\{*\}$, we have a fibration

$$
\Phi: L^{*}\left(\xi, \mathcal{U}_{G} ; *\right) \rightarrow B_{\mathrm{aut}_{G} F}
$$

with fibre $\operatorname{aut}_{G} F$. Therefore we recover

Corollary 2. If $F$ is compactly generated, then

$$
\pi_{i}\left(B_{\operatorname{aut}_{G} F}\right) \cong \pi_{i-1}\left(\operatorname{aut}_{G} F\right), \quad i \geq 1 .
$$

Corollary 3. If $B$ is a simply connected finite $C W$ complex and $F$ has the homotopy type of a simply connected finite type $C W$ complex, then the groups $\pi_{i}\left(\mathcal{L}_{1}(\xi)\right), i \geq 1$, are finitely generated.

Proof: Denote by $F_{0}$ the rationalisation of the space $F$. The induced $\operatorname{map} \pi_{n}\left(\operatorname{aut}_{1} F\right) \rightarrow \pi_{n}\left(\operatorname{aut}_{1}\left(F_{0}\right)\right)$ is finite to one for $n \geq 1([\mathbf{1 2},(5.4)])$. 
On the other hand, denoting by $M$ the Sullivan minimal model of $F$, we have a sequence of group isomorphisms $\pi_{n}\left(\operatorname{aut}_{1}\left(F_{0}\right)\right) \cong \pi_{n}\left(\left(\operatorname{aut}_{1}(F)\right)_{0}\right) \cong$ $\pi_{n}\left(\operatorname{aut}_{1}(M)\right)([\mathbf{1 2}, 3.11])$. As $\pi_{n}\left(\operatorname{aut}_{1}(M)\right)$ is finitely generated, the same is true for $\pi_{n}\left(\operatorname{aut}_{1}(F)\right)$ for $n \geq 1$. We now make use of the Federer spectral sequence $([\mathbf{7}])$ converging to $\pi_{*}\left(L\left(B, B_{\text {aut }_{1} F}, k\right)\right)$. It is easy to see that $E_{p, q}^{2}=H^{q}\left(B, \pi_{p+q}\left(B_{\text {aut }_{1} F}\right)\right)$ is finitely generated abelian so that $E_{p, q}^{\infty}$ is finitely generated abelian. Since an extension of finitely generated abelian groups is a finitely generated abelian group, the groups $\pi_{n}\left(L\left(B, B_{\text {aut }_{1} F}, k\right)\right)$ are finitely generated.

Consider now the evaluation map

$$
e: L\left(S^{n}, B_{\mathrm{aut}_{G} F}\right) \rightarrow B_{\mathrm{aut}_{G} F} .
$$

This is a Hurewicz fibration and the fibre is the space of based maps $L_{\bullet}\left(S^{n}, B_{\text {aut }_{G} F}\right)$. It results from $([\mathbf{2 2}$, Theorem 3.2]) that the homotopy exact sequence associated to this fibration is isomorphic to the exact sequence

$$
\begin{aligned}
& \rightarrow \pi_{i+1}\left(B_{\operatorname{aut}_{G} F}\right) \stackrel{[k,-]}{\longrightarrow} \pi_{n+i}\left(B_{\operatorname{aut}_{G} F}\right) \\
& \stackrel{T}{\longrightarrow} \pi_{i}\left(L\left(S^{n}, B_{\operatorname{aut}_{G} F}\right) ; k\right) \stackrel{e_{*}}{\longrightarrow} \pi_{i}\left(B_{\operatorname{aut}_{G} F}\right)
\end{aligned}
$$

where $[k,-]$ denotes the Whitehead bracket and $T=\tau \circ \pi_{*}(j)$ where $j$ is the canonical injection

$$
j: L_{\bullet}\left(S^{n}, B_{\text {aut }_{G} F}\right) \rightarrow L\left(S^{n}, B_{\text {aut }_{G} F}\right),
$$

and $\tau$ the natural isomorphism

$$
\pi_{n+i}(Y)=\left[S^{i} \wedge S^{n}, Y\right]=\pi_{i}\left(L_{\bullet}\left(S^{n}, Y\right)\right) \cong \pi_{i}\left(L_{\bullet}\left(S^{n}, Y\right), k\right), \quad i \geq 1 .
$$

The natural isomorphism

$$
\partial_{Y}: \pi_{i}(Y) \rightarrow \pi_{i-1}(\Omega Y)
$$

transforms the Whitehead product into the Samelson product, up to a sign, and $\pi_{*}(e)$ into $R: \mathcal{L}_{G}(\xi) \rightarrow \operatorname{Aut}_{G} F=G$. Then, using corollaries 1 and 2 above, we deduce the exact sequence of groups

$$
\pi_{1}\left(\operatorname{aut}_{G} F\right) \stackrel{\partial_{k}}{\longrightarrow} \pi_{n}\left(\operatorname{aut}_{G} F\right) \stackrel{\gamma}{\longrightarrow} \mathcal{L}_{G}(\xi) \stackrel{R}{\longrightarrow} G,
$$

with $\gamma=\partial_{L\left(S^{n}, B_{\text {aut }_{G} F}\right)} \circ T \circ \partial_{B_{\text {aut }}^{-1} F_{F}}$. Now by $([\mathbf{1 3}$, Theorem 2.2]), we know that the image of $R$ is precisely $G_{\alpha}$. 


\section{Proof of Theorem 3}

Let us consider the cochains $\mathcal{C}^{*}\left(L_{*}\right)$ on the differential graded Lie algebra $L_{*}$ defined in the introduction,

$$
\mathcal{C}^{*}\left(L_{*}\right)=\left(\wedge s\left(L_{*}^{\vee}\right), d\right)
$$

where $L_{*}^{\vee}$ denotes the graded vector space dual to $L$

$$
\left(L_{*}^{\vee}\right)^{i}=\operatorname{Hom}\left(L_{-i}, \mathbb{Q}\right) .
$$

By $\left(\left[\mathbf{2 0}\right.\right.$, section 11]), $\left(\wedge s L_{*}^{\vee}, d\right)$ is a (non minimal) model of $B_{\text {aut }_{G} F}$ when $G$ is a maximal subgroup of Aut $F$ acting unipotently on $H_{*}(F ; \mathbb{Q})$. Thus, if $F$ is a nilpotent compactly generated space, Corollary 2 together with $([\mathbf{2 0}$, Theorem 10.1]) give the isomorphism

$$
\pi_{i}\left(\operatorname{aut}_{G} F\right) \cong H_{i}\left(L_{*}, D\right), \quad i \geq 1 \text {. }
$$

If $L$ is a locally nilpotent Lie algebra over $\mathbb{Q}$, we denote by $\exp (L)$ the divisible group associated to $L$ by the Campbell-Hausdorff formula

$$
x \cdot y=x+y+\frac{1}{2}[x, y]+\cdots
$$

Let $G$ be a finitely generated torsion free nilpotent group. In $([\mathbf{1 4}])$, Malcev constructs a Lie algebra $L_{G}$ over the rationals such that $G$ naturally embeds into $\exp \left(L_{G}\right)$. The group $\hat{G}=\exp \left(L_{G}\right)$ is called the Malcev completion of $G([\mathbf{1 6}],[\mathbf{1 4}])$.

Let now $X$ be a nilpotent space. The action of $\pi_{1}(X)$ onto $\pi_{n}(X)$ can be described, modulo the isomorphism $\pi_{r}(X) \cong \pi_{r-1}(\Omega X)$, by the map

$$
\begin{gathered}
\mu: \pi_{0}(\Omega X) \times \pi_{n-1}(\Omega X) \rightarrow \pi_{n-1}(\Omega X), \\
\mu(g, \alpha)=g \cdot \alpha(t) \cdot g^{-1} .
\end{gathered}
$$

Such a space $X$ admits a 0-localization $X_{0}$, which satisfies $\pi_{1}\left(X_{0}\right)=$ $\widehat{\pi_{1}(X)}, \pi_{i}\left(X_{0}\right)=\pi_{i}(X) \otimes \mathbb{Q}, i \geq 2$. Moreover, the action of $\pi_{1}(X)$ on $\pi_{*}(X)$ induces an action of the Lie algebra $L_{\pi_{1}(X)}$ on $\pi_{n}(X) \otimes \mathbb{Q}$ which is given by the bracket in the Lie algebra $\pi_{*}(\Omega X) \otimes \mathbb{Q}([\mathbf{2}])$.

We now return to the particular case, $\Omega X=\operatorname{aut}_{G} F$. Let $\eta$ be a derivation that represents $\alpha$. We then have

$$
\exp \left(H_{0}\left(L_{*}, D\right)_{\eta}\right)=\widehat{G_{\alpha}}
$$




\section{Proof of Theorems 4 and 5}

The rational homotopy groups $\pi_{i}\left(L\left(B, B_{\operatorname{aut}_{G} F}, k\right)\right) \otimes \mathbb{Q}, i>1$ and the Malcev completion of the nilpotent group $\pi_{1}\left(L\left(B, B_{\operatorname{aut}_{G} F}, k\right)\right)$ can be computed by rational homotopy theory and more precisely by Haefliger's work on mapping spaces $([\mathbf{1 0}])$. In fact, if $f: S \rightarrow T$ is a continuous map between nilpotent finite type $\mathrm{CW}$ complexes, then there exists a complex $\left(D_{*}, \partial\right)$,

$$
D_{n}=\oplus_{p}\left[H^{p}(S ; \mathbb{Q}) \otimes \pi_{n+p}\left(T_{0}\right)\right]
$$

such that

(i) $H_{q}\left(D_{*}, \partial\right) \cong \pi_{q}(L(S, T ; f)) \otimes \mathbb{Q}$, for $q>1$.

(ii) $\left.H_{1}\left(D_{*}, \partial\right)=\pi_{1}(L \widehat{(S, T ; f})\right)$.

The differential $\partial$ depends on the map $f$ and the construction is described in $([\mathbf{1 0}],[\mathbf{8}])$.

Proof of Theorem 4: When $F$ is an homogeneous space $G=K / H$, with $\operatorname{rank} K=\operatorname{rank} H$, Shiga and Tezuka $([\mathbf{1 8}])$ prove that

$$
\pi_{2 r}\left(\operatorname{aut}_{G} F\right) \otimes \mathbb{Q}=0, r \geq 1 .
$$

This implies :

$$
D_{2 n+1}=\bigoplus_{2 p \leq 2 n+1} H^{2 p}(B ; \mathbb{Q}) \otimes \pi_{2 n+1+2 p}\left(B_{\mathrm{aut}_{G} F}\right)=0,
$$

and thus $\partial=0$. Therefore,

$$
\left\{\begin{array}{l}
\pi_{2 n}\left(L\left(B, B_{\operatorname{aut}_{G} F} ; k\right)\right) \otimes \mathbb{Q}=D_{2 n}, \quad n \geq 0 \\
\pi_{2 n+1}\left(L\left(B, B_{\operatorname{aut}_{G} F} ; k\right)\right) \otimes \mathbb{Q}=0
\end{array}\right.
$$

Now Corollary 1 implies that $\widehat{\mathcal{L}_{G}(\xi)}=0$. The rationalization $\left(L_{1}(\xi)\right)_{0}$ of $L_{1}(\xi)$ is a finite dimensional rational $\mathrm{H}$-space, with

$$
\begin{aligned}
& \pi_{2 n}\left(\left(L_{1}(\xi)\right)_{0}\right)=0 \\
& \pi_{2 n-1}\left(\left(L_{1}(\xi)\right)_{0}\right)=\bigoplus_{p \leq n} H^{2 p}(B ; \mathbb{Q}) \otimes \pi_{2 n+2 p}\left(B_{\text {aut } F}\right)
\end{aligned}
$$

This proves Theorem 4 .

Proof of Theorem 5: We now suppose that $\tilde{H}_{q}(B ; \mathbb{Z})$ is finite for $q \leq n$ and that $\pi_{q}(F)$ is finite for $q>n$. This implies that

$$
D_{1}=H^{0}(B ; \mathbb{Q}) \otimes \pi_{1}\left(\widehat{B_{\operatorname{aut}_{G}} F}\right) \cong \widehat{G},
$$


and

$$
D_{q} \cong H^{0}(B ; \mathbb{Q}) \otimes \pi_{q+1}\left(\operatorname{aut}_{G} F\right) \cong \pi_{q+1}\left(\operatorname{aut}_{G} F\right) \otimes \mathbb{Q}, \text { for } q>1 .
$$

In particular, $\hat{R}: \widehat{\mathcal{L}_{G}(\xi)} \rightarrow \hat{G}$ is injective, $L_{1}(\xi)$ is a connected space and the evaluation map

$$
e: L\left(B, B_{\text {aut }_{1} F} ; k\right) \rightarrow B_{\text {aut }_{1} F}
$$

is a rational homotopy equivalence. The commutativity of the following diagram together with Proposition 1 implies now that $L_{1}(\xi) \rightarrow$ aut $_{1} F$ is also a rational homotopy equivalence.

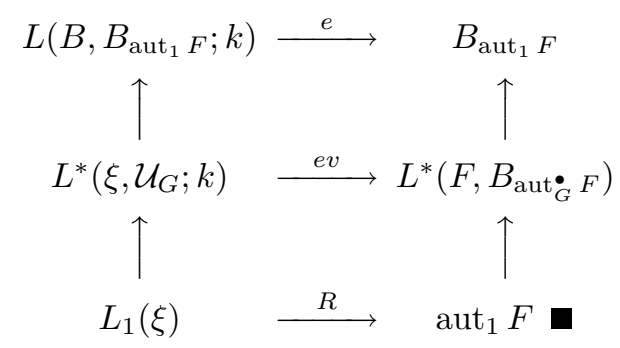

Using rational homotopy we can make explicit computations.

Proposition 2. Let $\xi: E \rightarrow B$ be a fibration with fibre $F$. We suppose that $B$ and $F$ are simply connected finite type $C W$ complexes and that there exists an integer $N$ such that $\pi_{>N}(F) \otimes \mathbb{Q}=0$, then

1) $\pi_{n}\left(L_{1}(\xi)\right)$ is a finite group for $n>N$.

2) We have isomorphisms

$$
\pi_{N}\left(L_{1}(\xi)\right) \otimes \mathbb{Q}^{\pi_{N}(R)} \longrightarrow \pi_{N}\left(\operatorname{aut}_{1}(F)\right) \otimes \mathbb{Q} \stackrel{\pi_{N}(e v)}{\longrightarrow} \pi_{N}(F) \otimes \mathbb{Q} .
$$

Proof: The rational homotopy groups of the space $\operatorname{aut}_{1}(F)$ are isomorphic to the homology groups of the space of derivations of the Sullivan minimal model of $F([\mathbf{2 0}])$. It is then clear that $\pi_{>N}\left(\operatorname{aut}_{1}(F)\right) \otimes \mathbb{Q}=0$ and that the evaluation map $e v: \operatorname{aut}_{1}(F) \rightarrow F$ induces an isomorphism on $\pi_{N}(-) \otimes \mathbb{Q}$. As $B$ is simply connected, this implies that the vector spaces $D_{n}$ are zero for $n>N$ and for $n=N-1$. Therefore we have the isomorphisms $\pi_{N}\left(L_{1}(\xi) \otimes \mathbb{Q} \cong D_{N}=H^{0}(B ; \mathbb{Q}) \otimes \pi_{N}\left(\operatorname{aut}_{1}(F)\right)\right.$. 


\section{References}

1. G. Allaud, On the classification of fibre spaces, Math. Z. 92 (1966), 110-125.

2. B. Cenkl And T. Porter, Malcev's completion of a group and differential forms, J. of Differential Geometry 15 (1980), 531-542.

3. G. Cooke, Replacing homotopy actions by topological actions, Trans. Amer. Math. Soc. 237 (1978), 391-406.

4. A. Dold, Partitions of unity in the theory of fibrations, Ann. of Math. 78 (1963), 223-255.

5. E. Dror, W. Dwyer and D. Kan, Self homotopy equivalences of virtually nilpotent spaces, Comment. Math. Helvetici 56 (1981), $599-614$.

6. E. Dror And A. ZABrodsky, Unipotency and nilpotency in homotopy equivalences, Topology 18 (1979), 187-197.

7. H. Federer, A study of function spaces by spectral sequences, Trans. Amer. Math. Soc. 82 (1956), 340-361.

8. Y. FÉLIX AND J.-C. Thomas, The monoid of self-homotopy equivalences of some homogeneous spaces, Expositiones Mathematicae 12 (1994), 305-322.

9. D. H. Gottlieb, On fibre spaces and the evaluation map, Ann. of Math. 87 (1968), 42-55.

10. A. Haefliger, Rational homotopy of the space of sections of a nilpotent bundle, Trans. Amer. Math. Soc. 273 (1977), 173-199.

11. S. HAlPerin, Lectures on minimal models, Mémoire Soc. Math. France 9/10 (1983).

12. P. Hilton, G. Mislin and J. Roitberg, "Localization of Nilpotent Groups and Spaces," North Holland Mathematics Studies 15, North Holland, 1975.

13. S. T. Hu, Concerning the homotopy groups of the components of the mapping spaces $Y^{S^{p}}$, Indag. Math. 8 (1946), 623-629.

14. A. I. MALCEV, On a class of homogeneous spaces., Izv. Akad. Nauk. SSSR, Ser. Math. 13 (1949), 9-39; English transl. Amer. Math. Sco. Transl. 9 (1962), 276-307.

15. H. Oshima and K. Tsukiyama, On the group of Equivariant Self Equivalences of Free actions, Publ. RIMS Kyoto Univ. 22 (1986), 905-923.

16. D. Quillen, Rational homotopy theory, Annals of Math. 90 (1969), 205-295. 
17. F. Quinn, Nilpotent spaces and actions of finite groups, Houston J. Math. 4 (1978), 239-248.

18. H. Shiga And M. Tezuka, Rational fibrations, homogeneous spaces with positive Euler characteristics and jacobians, Annales Inst. Fourier 37 (1987), 81-106.

19. E. Spanier, "Algebraic Topology," Mc. Graw Hill, New York, 1966.

20. D. Sullivan, Infinitesimal computations in topology, Publ. I.H.E.S. 47 (1977), 269-331.

21. K. Tsukiyama, On the group of fibre homotopy equivalences, $H i$ roshima Math. J. 12 (1982), 349-376.

22. G. Whitehead, On products in homotopy theory, Ann. of Math. 47 (1946), 460-475.

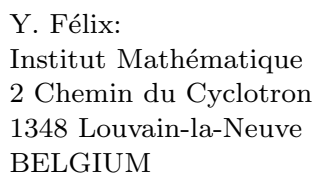

\author{
J. C. Thomas: \\ U.F.R. de Mathématiques \\ Université de Lille-Flandres-Artois \\ F-59655 Villeneuve d'Ascq
}

FRANCE

Primera versió rebuda el 29 d'Abril de 1994, darrera versió rebuda el 18 de Gener de 1995 